\title{
Diagnosis and treatment of post-traumatic stress disorder during the COVID-19 pandemic
}

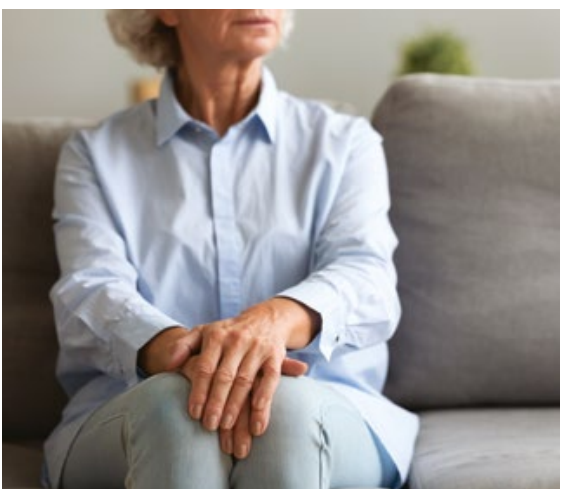

CPD

John Cooper, Andrea J Phelps, Chee $\mathrm{H} \mathrm{Ng}$, David Forbes

\section{Background \\ The COVID-19 pandemic has caused unprecedented stress globally, and the associated medical and health-related traumatic experiences pose significant risks for the development of post- traumatic stress disorder (PTSD), and the exacerbation of pre-existing PTSD, among patients, general practitioners (GPs) and healthcare staff.}

\section{Objective}

The aim of this article is to provide guidance to GPs and healthcare staff working in Australia about the diagnosis and treatment of both newly developed and pre-existing PTSD in the COVID-19 context. Case studies are presented; the authors discuss whether pandemic-related PTSD is different to PTSD caused by different types of traumatic exposure, and the associated implications for treatment.

\section{Discussion}

The role of GPs in the management of PTSD during the COVID-19 pandemic remains central, involving early detection, assessment and referral. Moreover, health professionals are not immune to the mental health effects of the pandemic and are encouraged to maintain their wellbeing and to seek professional treatment if needed.
THE COVID-19 PANDEMIC is causing many and varied stressors globally. Beyond the direct impact of the infection, stress can arise from uncertainty, loss of control, isolation, work demands, burnout and fatigue, unemployment and financial strain, among other concerns.

These more general stressors affect health in non-specific ways. While most people will be resilient and cope, those with vulnerable pre-existing physical and mental disorders or vulnerabilities are likely to experience exacerbations of these conditions.

The general stressors arising from the pandemic can be associated with a wide range of mental health sequelae. This is reflected in results of an online survey of 5070 Australian adults conducted in March and April 2020, which showed significantly elevated levels of anxiety, depression and stress. ${ }^{1}$ This article will, however, focus on the more severe type of pandemic-related stress consistent with traumatic stress where resources are overwhelmed and lives are threatened and lost. The diagnosis of post-traumatic stress disorder (PTSD) as defined in the Diagnostic and statistical manual for mental disorders, 5 th edition (DSM-5), requires exposure to a traumatic event, which is defined as 'actual or threatened death, serious injury, or sexual violence'. ${ }^{2}$
PTSD is one of the most common disorders following exposure to a traumatic event. The Australian National Mental Health and Wellbeing Survey ${ }^{3}$ reported that approximately $10 \%$ of people who have experienced a potentially traumatic event will develop PTSD at some stage in their lives. ${ }^{4}$ PTSD is not the only mental disorder that can follow a traumatic event. Other common post-traumatic conditions include depression, anxiety disorders and substance abuse. PTSD is, however, considered the 'signature' post-traumatic mental disorder and, as such, is the focus of this article. Importantly, PTSD is the second most prevalent mental disorder, after depression, with $>4.4 \%$ of Australians meeting criteria for the diagnosis in any 12-month period. ${ }^{5}$ Evidence indicates that aforementioned stressors, which occur subsequent to a potentially traumatic event, can have a significant role in the development and maintenance of PTSD. ${ }^{6}$ Hence, the COVID-19 pandemic can both cause PTSD and, through the stressors it generates, significantly exacerbate and perpetuate a pre-existing PTSD.

\section{Assessment considerations}

Currently there are two distinct diagnostic systems with differing criteria for PTSD. The DSM-5 has a more expansive set of 
criteria including 20 different symptoms across the domains of re-experiencing, avoidance, negative cognitions and moods, and hyperarousal. The International classification of diseases, 11 th revision, (ICD-11) has only six symptoms across three domains: re-experiencing, avoidance and hyperarousal. ${ }^{7}$ The diagnostic criteria for ICD-11 PTSD are listed in Box 1. ICD-11 also has diagnostic criteria for complex PTSD, the symptoms of which overlap to some extent with the extended criteria of DSM-5 PTSD.

The assessment issues are further illustrated in Cases 1 and 2.

\section{CASE 1}

Fred is a married tradesman aged 50 years and a father of three. His wife treated him to a cruise to celebrate his fiftieth birthday. During the cruise they both contracted COVID-19 and were taken from the ship into hotel quarantine. Fred was febrile, became progressively short of breath and felt as if he were drowning. He was taken by ambulance to hospital and admitted to the intensive care unit (ICU). Prior to the decision to be placed on a ventilator, Fred believed he was dying and made mental preparations.

Fred was discharged from ICU after 10 days and, while still in the medical ward, became increasingly anxious. $\mathrm{He}$ feared falling asleep, as he was worried that he would not wake up. He was highly sensitive and attuned to his breathing patterns and pulse. When confronted by the medical team performing their rounds, he developed sweatiness, flushing and palpitations. In the following months, Fred worried about his children, fearing they would also contract the virus. He became obsessional about safety, insisting they isolate and avoid any risk of infection. Fred felt depressed and pessimistic, and he stopped reading newspapers and watching the evening news.

This case shows how medical and healthrelated stressors can be traumatic. COVID-19 fatalities are also potentially traumatic for surviving family members and health professionals.
Australia's public health response has spared us so far from dire international experiences where health systems have been overwhelmed, with insufficient ICU beds and ventilators, and resources have been rationed. It is clear from these horrific reports that clinicians and health administrators have been in predicaments where they make life-or-death decisions about patients who qualify for intensive treatment and those who may die from lack of treatment. The impact of these traumatic situations can be viewed through the lens of moral injury. A recent survey during the COVID-19 pandemic found that the prevalence of a moderate-to-severe stress level was $>60 \%$ among frontline healthcare workers. ${ }^{8}$ More information on moral injury and COVID-19, and other useful resources for healthcare professionals, are listed in Table 1.

\section{CASE 2}

Patricia is a retired public servant aged 63 years who lives alone with her beloved dog. Patricia has been diagnosed with complex PTSD arising from sustained childhood physical and sexual abuse perpetrated by her adoptive father. Following migration to Australia, she was able to distance herself from her adoptive family, graduate from university and have a successful professional career. Over the two decades leading into her mid-fifties, she was in a relationship with a violent and controlling man. On many occasions she feared for her life.

At the start of the COVID-19 pandemic, Patricia welcomed the restrictions and isolation imposed by public health measures. She had a legitimate reason to avoid social and public places that historically triggered her traumatic memories and associated extreme distress.

As the months dragged on, Patricia's anxiety, insomnia, nightmares and fear of contracting the virus flared as she became housebound. She simultaneously wanted to die and was morbidly fearful of becoming ill with COVID-19.

Pre-pandemic, Patricia was least anxious when alone at home with her dog but would force herself to go to the local shops and to yoga classes, and to socialise with friends. The physical distancing and isolation rules reinforced and legitimised her avoidance, and this eventually increased her overall anxiety (and avoidance). Pre-pandemic she was planning to volunteer with a migrant support network. She now believes this will never happen.

Case 2 shows the possible impacts the pandemic can have on people with pre-existing PTSD. Many of the functional impairments caused by chronic PTSD occur through the mechanism of avoidance. Triggers to distressing

\section{Box 1. International classification of diseases, 11th revision, (ICD-11) criteria for post-traumatic stress disorder ${ }^{7}$}

'Post-traumatic stress disorder (PTSD) is a disorder that may develop following exposure to an extremely threatening or horrific event or series of events. It is characterized by all of the following:

1. re-experiencing the traumatic event or events in the present in the form of vivid intrusive memories, flashbacks, or nightmares. These are typically accompanied by strong or overwhelming emotions, particularly fear or horror, and strong physical sensations;

2. avoidance of thoughts and memories of the event or events, or avoidance of activities, situations, or people reminiscent of the event or events; and

3. persistent perceptions of heightened current threat, for example as indicated by hypervigilance or an enhanced startle reaction to stimuli such as unexpected noises.

The symptoms persist for at least several weeks and cause significant impairment in personal, family, social, educational, occupational or other important areas of functioning.'

Reproduced with permission from World Health Organization, International statistical classification of diseases and related health problems, 11th edn, Geneva, CH: WHO, 2019. 


\section{Table 1. COVID-19 mental health resources for healthcare professionals}

\begin{tabular}{lll}
\hline Organisation & Name of document & Website \\
\hline $\begin{array}{l}\text { Phoenix Australia - Centre for } \\
\text { Posttraumatic Mental Health }\end{array}$ & $\begin{array}{l}\text { Moral stress amongst healthcare } \\
\text { workers during COVID-19: A guide } \\
\text { to moral injury }\end{array}$ & $\begin{array}{l}\text { www.phoenixaustralia.org/wp-content/uploads/2020/07/ } \\
\text { Moral-Stress-Healthcare-Workers-COVID-19-Guide-to-Moral- } \\
\text { Injury.pdf }\end{array}$ \\
\hline Black Dog Institute & $\begin{array}{l}\text { Depression in health care workers } \\
\text { during COVID-19 }\end{array}$ & $\begin{array}{l}\text { www.blackdoginstitute.org.au/wp-content/uploads/2020/05/ } \\
\text { Depression-in-Health-Care-Workers-during-COVID-19-1.pdf }\end{array}$ \\
\hline $\begin{array}{l}\text { Australian Government } \\
\text { Department of Health }\end{array}$ & $\begin{array}{l}\text { Looking after your mental health } \\
\text { during coronavirus (COVID-19) } \\
\text { restrictions }\end{array}$ & $\begin{array}{l}\text { www.health.gov.au/news/health-alerts/novel-coronavirus- } \\
\text { 2019-ncov-health-alert/ongoing-support-during-coronavirus- } \\
\text { covid-19/looking-after-your-mental-health-during-coronavirus- } \\
\text { covid-19-restrictions }\end{array}$ \\
\hline $\begin{array}{l}\text { Australian Psychources } \\
\text { Society }\end{array}$ & $\begin{array}{l}\text { Frontline workers and COVID-19 } \\
\text { Tips for coping with COVID-19 anxiety }\end{array}$ & $\begin{array}{l}\text { www.psychology.org.au/for-the-public/Psychology-topics/ } \\
\text { COVID-19-Australians }\end{array}$ \\
\hline
\end{tabular}

traumatic memories can be both internal (thoughts, feelings, physical sensations) and external (places, people, other stimuli). Over time, and often over many years, the triggers can generalise and seem less obviously connected to the original trauma, as can the patterns of avoidance. In these situations, post-traumatic avoidance can take on similar characteristics to agoraphobia or social phobia. In addition, the negative cognitive biases associated with PTSD can accentuate the anxiety and distress about the risk of contagion.

There are questions of whether pandemic-related PTSD is substantially different to PTSD caused by different types of trauma exposure and, if so, whether this affects treatment. Effective management of PTSD begins with a thorough individual assessment, requires evidence-based approaches and is bedded in engagement, rapport and trust. Engagement, rapport and trust are better achieved when there is awareness and sensitivity to the patient's experiences and background and the nature of their trauma. For example, military personnel and veterans engage more readily when their service and deployment experiences are understood, ${ }^{9}$ and victims of sexual assault will understandably take longer to develop trust. ${ }^{10}$ In the same way, the unique nature of traumatic experiences that arise in the context of a pandemic needs to be understood to promote the patient's engagement, rapport and trust.
The threat posed by a pandemic is invisible, and this engenders uncertainty. It disrupts a sense of control and self-determination. The stress induced can be both acute (panic, sense of crisis) and chronic (fatigue, burnout, demoralisation). There is a very personal element to this traumatic stressor as viral transmission occurs between people in close proximity, usually loved ones, friends and colleagues. This can lead to feelings of guilt and shame. Stigmatisation and alienation are common during the COVID-19 pandemic and can exacerbate the adverse psychological impact. ${ }^{11}$

Isolation and physical distancing are some of the primary public health mechanisms for controlling the spread of the virus. These life-saving mechanisms are antithetical to the mechanisms of social connection and support that are vital for protecting against the development of post-traumatic mental health disorders.
Unemployment and financial stress are also adverse consequences of the pandemic experience for many people. These are well-established risk factors for a range of mental health disorders, including PTSD.

\section{Treatment considerations}

When it comes to the management of PTSD, the role of GPs is central. ${ }^{12,13}$

This role includes:

- provision of initial support and monitoring (eg psychological first aid [PFA] - refer to Box 2)

- early detection, initial assessment and supportive management

- use of initial pharmacotherapy

- appropriate and timely referral for specialist treatment, for example when the symptoms are persisting (for more than four weeks), severe or complex, associated with risk, or significantly impairing function

\section{Box 2. Psychological first aid ${ }^{17,18}$}

Australian ${ }^{17}$ and international ${ }^{18}$ guidelines recommend providing assistance consistent with the principles and actions of psychological first aid (PFA) to people who have experienced a traumatic event.

PFA seeks to:

- reduce initial distress

- address basic needs (eg comfort, information, practical and emotional support)

- promote adaptive coping (eg problem solving)

- encourage engagement with existing social supports. 
- support of family and carers

- crisis assistance

- management of comorbid medical conditions

- maintenance treatment for chronic conditions.

A comprehensive account of evidencebased treatment of new and established cases of PTSD can be found in the most recent edition of the 2020 National Health and Medical Research Councilapproved Australian guidelines for the prevention and treatment of acute stress disorder, posttraumatic stress disorder and complex PTSD. ${ }^{14}$

\section{Psychological therapies}

The available evidence for both acute and established PTSD leads to similar recommendations, which indicate that trauma-focused psychological therapies are the recommended first-line treatments in both circumstances. This is a conditional recommendation in the case of acute PTSD (the intervention should be provided to most people, in preference to doing nothing) and a strong recommendation in the case of established PTSD (the intervention should be provided to all or almost all people in all or almost all circumstances). There is also a 'strong recommendation' for the use of a stepped/collaborative care model with acute PTSD. ${ }^{14}$

When referring patients for evidencebased psychological treatment of PTSD, it is useful to have a good working knowledge of what trauma-focused psychological treatment entails. ${ }^{12}$ Patients often appreciate general counselling, which involves dealing with daily issues and stresses and talking around, but not about, the trauma; however, these techniques do not improve core symptoms of PTSD. Efficacious trauma-focused interventions include therapies such as prolonged exposure, eye movement desensitisation and reprocessing (EMDR), cognitive processing therapy and cognitive therapy. The common factors in these therapies that are considered to be the 'potent' elements are strategies that: - enable the patient to confront the distressing traumatic memories
- manage the accompanying avoidance responses

- facilitate reduction and management of the associated arousal

- modify unhelpful interpretations relating to what the event means about themselves, others or the world - that are impeding recovery.

These evidence-based psychological treatments are time-limited and usually involve 8-12 sessions, although more sessions may be required for complex presentations or repeated traumatic experiences. Therapies such as prolonged exposure may also require longer sessions (ie 90 minutes) to ensure appropriate management of distress. When choosing a psychologist or a trained therapist for patient referral, it is recommended that the GP enquire about their experience in treating PTSD with trauma-focused cognitive behavioural therapy (TF-CBT) or EMDR.

\section{Pharmacotherapy}

Pharmacological treatment remains a second-line option for PTSD, with antidepressant medications having the strongest evidence base. ${ }^{14}$ Antidepressant medications with the strongest evidence base include selective serotonin reuptake inhibitors (sertraline, paroxetine or fluoxetine) and venlafaxine, and they should be considered for people who: ${ }^{12}$

- are unwilling to seek or do not have access to the preferred first-line psychological treatment

- have a comorbid depression or other symptoms that require pharmacological treatment

- are in situations that are not sufficiently stable (eg ongoing domestic violence)

- have not benefited from traumafocused psychological treatment. There is evidence that the required doses are higher when treating PTSD, compared with uncomplicated depression. When there is a good response to an antidepressant, a course of treatment lasting at least 12 months reduces rates of relapse. When second- and third-line pharmacotherapy options need to be considered because of non-response or side effects, other newer generation antidepressants such as other selective serotonin and noradrenaline reuptake inhibitors or mirtazapine are recommended. ${ }^{15}$

Poor response to initial treatments, complex comorbidities, safety concerns and patient request should lead to referral for psychiatric assessment. Additional treatments such as tricyclic antidepressants (TCAs), monoamine oxidase inhibitors, atypical antipsychotics, mood stabilisers and benzodiazepines may need to be considered, but clinicians and patients need to be aware of their limited evidence base and their associated risks (eg metabolic syndrome with atypical antipsychotics; abuse and dependency with benzodiazepines; overdose with TCAs).

\section{Other treatment issues}

Patients with chronic forms of PTSD have been adversely affected by the pandemic through both chronic stress and, in some cases, neglect of important maintenance treatment. The early introduction of telehealth has helped to mitigate some of these concerns. Prior to this pandemic, there was a growing evidence base for using this medium to provide psychological treatment for patients with PTSD. With the support of Medicare Benefits Schedule rebates, telehealth has reduced the likelihood of patients withdrawing from treatment with their GP, psychologist or psychiatrist.

Any concern a GP has about exacerbating or reinforcing the adverse impact of trauma in their patients can be alleviated through a trauma-informed approach. This involves:

- thinking about whether a patient has experienced trauma

- being aware of the impacts of trauma

- allowing the patient to control the amount of information they disclose

- providing appropriate information and education to the patient.

Importantly, health professionals are not immune to the mental health effects of the pandemic. PTSD affects GPs and other health professionals at rates above the general population. ${ }^{16}$ The diverse stressors associated with the pandemic will exacerbate PTSD in affected doctors just as 
it will in their affected patients. Exhaustion, burnout and sleep deprivation accumulate as risk factors, increasing vulnerability to serious mental health problems such as PTSD and depression. Medical staff on the frontline - general practice, emergency departments, medical wards and ICUs - are exposed to life-threatening, potentially traumatic events, and some will develop PTSD. It is important for healthcare workers to maintain their wellbeing and seek appropriate professional treatment if needed. ${ }^{13}$

\section{Conclusion}

The COVID-19 crisis poses significant risks for the development of PTSD and the exacerbation of existing PTSD within patients seen in general practice. The circumstances also pose a significant challenge for GPs and their staff, who must maintain their own health and wellbeing in the context of the physical threat, the consequential stressors including financial and social distancing related, and potentially challenging medical triaging decisions.

\section{Authors}

John Cooper MBBS, MPM, FRANZCP Consultant Psychiatrist, Phoenix Australia Centre for Posttraumatic Mental Health and Department of Psychiatry, The University of Melbourne, Vic. cooperja@unimelb.edu.au

Andrea J Phelps BA (Hons), MPsych (Clin), PhD, Deputy Director and Associate Professor, Phoenix Australia Centre for Posttraumatic Mental Health and Department of Psychiatry, The University of Melbourne, Vic

Chee H Ng MBBS, MMed (Psych), MD, FRANZCP, Healthscope Chair of Psychiatry, The Professorial Unit, The Melbourne Clinic, Vic; Professor of Psychiatry, The University of Melbourne, Vic; Director, WHO Collaborating Centre in Mental Health, St Vincent's Hospital, Vic

David Forbes BA (Hons), M.A (Clin Psych), PhD, Director and Professor, Phoenix Australia Centre for Posttraumatic Mental Health and Department of Psychiatry, The University of Melbourne, Vic

Competing interests: $\mathrm{CHN}$ reports travel support and honorarium for delivery of a presentation from Lundbeck and Pfizer, as well as travel and honorarium for consulting services from Janssen, outside of the submitted work.

Funding: None.

Provenance and peer review: Commissioned, externally peer reviewed.

\section{References}

1. Newby J, O'Moore K, Tang S, Christensen $\mathrm{H}$, Faasse K. Acute mental health responses during the COVID-19 pandemic in Australia. PLoS One 2020;15(7):e0236562. doi: 10.1371/journal. pone.0236562.

2. American Psychiatric Association. Diagnostic and statistical manual of mental disorders, 5 th edn. Arlington, VA: APA, 2013.

3. Australian Bureau of Statistics. National survey of mental health and wellbeing: Summary of results. Canberra, ACT: ABS, 2018. Available at www.abs.gov.au/statistics/health/mental-health/ national-survey-mental-health-and-wellbeingsummary-results/latest-release [Accessed 14 October 2020]

4. Creamer M, Burgess P, McFarlane A. Posttraumatic stress disorder: Findings from the Australian national survey of mental health and well-being. Psychol Med 2001;31(7):1237-47. doi: 10.1017/s0033291701004287.

5. McEvoy PM, Grove R, Slade T. Epidemiology of anxiety disorders in the Australian general population: Findings of the 2007 Australian national survey of mental health and wellbeing. Aust N Z J Psychiatry 2011;45(11):957-67. doi: 10.3109/00048674.2011.624083.

6. Bryant RA, Gibbs L, Gallagher $\mathrm{HC}$, et al. Longitudinal study of changing psychological outcomes following the Victorian Black Saturday bushfires. Aust N Z J Psychiatry 2018;52(6):542-51. doi: $10.1177 / 0004867417714337$.

7. World Health Organization. International statistical classification of diseases and related health problems. 11th edn. Geneva, CH: WHO, 2019.

8. Tian T, Meng F, Pan W, et al. Mental health burden of frontline health professionals treating imported patients with COVID-19 in China during the pandemic. Psychol Med 2020;1-2. doi: 10.1017/ S0033291720002093.

9. Phoenix Australia - Centre for Posttraumatic Mental Health. Specific populations and trauma types: Military and ex-military personnel. Australian guidelines for the prevention and treatment of acute stress disorder, posttraumatic stress disorder and complex PTSD. Melbourne, Vic: Phoenix Australia, 2020.

10. Phoenix Australia - Centre for Posttraumatic Mental Health. Specific populations and trauma types: Sexual assault. Australian guidelines for the prevention and treatment of acute stress disorder, posttraumatic stress disorder and complex PTSD. Melbourne, Vic: Phoenix Australia, 2020.

11. Zhai Y, Du X. Loss and grief amidst COVID-19: A path to adaptation and resilience. Brain Behav Immun 2020;87:80-81. doi: 10.1016/j.bbi.2020.04.053.

12. Cooper J, Metcalf O, Phelps A. PTSD - An update for general practitioners. Aust Fam Physician 2014;43(11):754-57.

13. Wade D, Howard A, Fletcher S, Cooper J, Forbes D. Early response to psychological trauma: What GPs can do. Aust Fam Physician 2013;42(9):610-14.

14. Phoenix Australia - Centre for Posttraumatic Mental Health. Australian guidelines for the prevention and treatment of acute stress disorder, posttraumatic stress disorder and complex PTSD. Melbourne, Vic: Phoenix Australia, 2020.

15. Baldwin DS, Anderson IM, Nutt DJ, et al. Evidence-based pharmacological treatment of anxiety disorders, post-traumatic stress disorder and obsessive-compulsive disorder: A revision of the 2005 guidelines from the British Association for Psychopharmacology. J Psychopharmacol 2014;28(5):403-39. doi: $10.1177 / 0269881114525674$.
16. Sendler DJ, Rutkowska A, Makara-Studzinska M How the exposure to trauma has hindered physicians' capacity to heal: Prevalence of PTSD among healthcare workers. Eur J Psychiatry 2016;30(4):321-34.

17. Australian Red Cross and Australian Psychological Society. Psychological first aid: An Australian guide to supporting people affected by disaster. Carlton, Vic: Australian Red Cross, 2013.

18. Brymer M, Jacobs A, Layne C, et al. Psychological first aid: Field operations guide. Los Angeles, CA: NCTSN, 2006. 